\title{
APRENDIZAJE BASADO EN PROYECTOS EN EL ÁMBITO UNIVERSITARIO: UNA EXPERIENCIA DE INNOVACIÓN METODOLÓGICA EN EDUCACIÓN
}

\author{
María Fernández-Cabezas \\ Facultad de Ciencias de la Educación \\ Universidad de Granada \\ mariafc@ugr.es \\ https://doi.org/10.17060/ijodaep.2017.n1.v2.939
}

Fecha de Recepción: 6 Marzo 2017

Fecha de Admisión: 1 Abril 2017

\section{RESUMEN:}

El presente trabajo presenta una experiencia de innovación metodológica en el área de educación, concretamente en los Grados de Pedagogía, Educación Social y Educación Infantil. La metodología llevada a cabo es el Aprendizaje basado en proyectos, el cual pretende que el alumno relacione los contenidos conceptuales vistos en clase con la realidad del mundo que le rodea, y sobre todo con su futuro profesional, siendo así el estudiante el protagonista de su aprendizaje y el docente el guía del mismo, eje fundamental de la filosofía que subyace al Espacio Europeo de Educación Superior.

La metodología llevada a cabo se centra en los grupos que forman los alumnos, de 506 personas, grupos que trabajarán en el proyecto a lo largo de todo el cuatrimestre. En un primer momento, decidirán el tema en el que se van a centrar, diseñando a partir de este momento, un proyecto de intervención real. El profesor les hace un seguimiento mediante tutorías, y evalúa de forma continua su trabajo.

Para evaluar los logros obtenidos en el proceso de aprendizaje del alumnado, se utilizaron dos instrumentos, uno destinado a comprobar la evolución de la voluntad de trabajo de los estudiantes reflexionando ellos mismos sobre su desempeño en el grupo y sobre el de sus iguales, y otro centrado en valorar el nivel de satisfacción del alumnado con la metodología. Los resultados son altamente positivos, correlacionando con diferentes investigaciones previas en las que se han realizado trabajos similares, comprobando que aumenta el rendimiento, la motivación, y la capacidad de trabajo en grupo del alumnado.

Palabras clave: Aprendizaje basado en proyectos, Ciencias de la Educación, grupos de trabajo 


\section{APRENDIZAJE BASADO EN PROYECTOS EN EL ÁMBITO UNIVERSITARIO: UNA EXPERIENCIA DE INNOVACIÓN METODOLÓGICA EN EDUCACIÓN}

ABSTRACT:

The present work presents an experience of methodological innovation in the area of education, concretely in the degrees of Pedagogy, Social Education and Preschool Education. The methodology carried out is the Project-based Learning, which aim is that the student relates the conceptual contents seen in class to the reality of the world and especially with his professional future, being the student the protagonist of his learning and the teacher the guide of the same one, fundamental idea of the philosophy of the European Space of Higher Education.

The methodology carried out centres on the workgroups that form the students, of five or six persons. Groups that will be employed at the project along the whole subject. In the first moment, they will decide the topic in which they are going to centre on the project, designing from this moment, a project of real intervention. The teacher does a follow-up to them by means of tutorships, and a continuous assessment of their work.

To evaluate the achievements obtained in the learning of the students, two instruments were in use, one destined to verifying the evolution of the will of work of the students thinking they themselves about his performance in the group and about that of his mates, and other one centred in valuing the level of satisfaction of the student body with this methodology. The results are highly positive, correlating with different previous investigations in which similar works have been realized, verifying that it increases the performance, the motivation, and the capacity of work in group of the students.

Key words: Project-based learning, Sciences of education, workgroups

\section{INTRODUCCIÓN}

En el marco del Espacio Europeo de Educación Superior e inmersos en el momento social y educativo en el que nos encontramos, es necesario un cambio de perspectiva metodológica en la educación universitaria. Tradicionalmente la educación se ha centrado fundamentalmente en la enseñanza, y con ella en el profesor como eje del proceso de enseñanza-aprendizaje. Ahora, ese cambio conlleva el darle al alumnado un mayor protagonismo, poniendo todos los esfuerzos en su aprendizaje, relevando el rol del docente al de guía, orientador. Así, las teorías más conductistas y cognitivistas, dejan paso a las constructivistas, desarrollando en el alumnado un aprendizaje significativo (Carrasco, Donoso, Duarte-Atoche, Hernández y López, 2015; Muñoz y Díaz, 2009; Navarro, Pertegal, Gil, González y Jimeno, 2011), en el que se integran los conocimientos que ya posee con los nuevos que debe adquirir, haciendo que lo aprendido perdure en el tiempo. No es tan importante lo que el alumno sepa en un momento determinado, sino aquello que pueda llegar a saber, lo que se relaciona muy estrechamente con su capacidad de aprendizaje (Estruch y Silva, 2006). Más importante que aprender contenidos es el aprender a aprender, fomentando un aprendizaje continuo (Reverte, Gallego, Molina y Satorre, 2006; Taboada, Touriño y Doallo, 2010).

Este cambio de perspectiva conlleva una mayor relación con el futuro laboral y profesional de los estudiantes, quienes deberán desarrollar una serie de habilidades, competencias y actitudes complementarias al dominio de aquellos conocimientos específicos de las diferentes materias, siendo muy valiosas metodologías centradas en la investigación-acción (Muñoz y Díaz, 2009; Nourdine y Bemposta, 2008).

El Aprendizaje basado en proyectos (ABPr en adelante), es una metodología activa que surge en los 70, en diversos ámbitos universitarios relacionados con la pedagogía (Carrasco y cols., 2015), concretamente en la Universidad de McMaster (Canadá), para combatir un problema de desmotivación en los estudiantes (Nourdine y Bemposta, 2008). En dicha metodología, el alumno se implica en un proceso de investigación de manera relativamente autónoma que culmina con un proyecto 
final, los cuales materializan oportunidades de aprendizaje en el aula, estando relacionados con la realidad social. El alumno es el responsable de su propio aprendizaje, lo cual hace que tenga una mayor motivación, constatando la aplicación práctica de lo trabajado y desarrollando valores relacionados con su práctica profesional (Taboada, Toruiño y Doallo, 2010), a la vez que adquiere un mayor dominio de procedimientos y conceptos. Además, permite desarrollar actividades más acordes con las necesidades del alumnado, adaptándose al ritmo de cada uno de ellos relacionando lo aprendido en clase con la realidad que les rodea, siendo una herramienta muy útil en atención a la diversidad (Muñoz y Díaz, 2009).

El profesor debe crear un ambiente óptimo de aprendizaje guiando el proceso, alentando el uso de las estrategias metacognitivas y reforzando los esfuerzos tanto individuales como grupales, haciendo un seguimiento minucioso del diseño de los proyectos. Debe dar un feedback y realizar una evaluación a nivel grupal del aprendizaje adquirido por cada estudiante. Debe motivar, dinamizar los grupos, ser empático y tener habilidades de comunicación para fomentar las relaciones con el alumnado. Por su parte, los estudiantes han de tener una actitud activa, de investigación, saber planificarse, aunar e interrelacionar conocimientos, trabajar con los miembros de su grupo en la misma dirección, saber proyectar en la realidad los conocimientos adquiridos y presentárselos a sus compañeros.

Un aspecto muy positivo es el trabajo en pequeños grupos, de 506 personas, en los que el alumno debe aprender y fomentar habilidades comunicativas, interpersonales, desarrollando la autorregulación y respetando las contribuciones que realicen sus compañeros. Todos ellos tienen responsabilidad sobre las decisiones que se tomen y el trabajo conjunto depende del esfuerzo individual, integrando habilidades de colaboración para construir su conocimiento (Sánchez y Vidal, 2013). Aprenden a aprender y a que sus compañeros aprendan, retroalimentándose, superándose y aprendiendo de los errores, de esta forma aumentan su autoestima, sintiéndose orgullosos de su trabajo (Navarro, y cols, 2011). Valores todos ellos necesarios en su futuro profesional.

El aprendizaje basado en proyectos conlleva muchos beneficios, entre otros el que se posibilite una retroalimentación y evaluación continua por parte del profesor, el establecer un cronograma de actividades que evidencien el progreso de los diferentes grupos, generar un espacio de reflexión por parte del estudiante, prepararlo para su futuro profesional y laboral, incrementando la motivación y la implicación tanto de alumnos como de profesores. Aumenta sus habilidades sociales y de comunicación, mejora la resolución de problemas y aprenden a trabajar en grupo (Collazos, 2009). Además, promueve el pensamiento creativo y la toma de decisiones, fomentando un mayor rendimiento académico. Teniendo todo lo anterior presente se puede afirmar que el ABPr facilita el aprendizaje de nuevos conocimientos y permite aplicar los ya adquiridos, desarrolla habilidades transversales, entre ellas la planificación, redacción, comunicación, así como la responsabilidad para afrontar una situación real (Carraco y cols., 2015).

Son muchas las universidades que han llevado a cabo la implementación de nuevas metodologías, entre ellas el ABPr, y diferentes son las asignaturas en las que su puesta en práctica ha tenido resultados satisfactorios, como es en la Universidad Europea de Madrid en la materia de robótica (Nourdine y Bemposta, 2008), en Ingeniería informática en la Universidad de Alicante (Reverte, Gallego, Molina y Satorre, 2006), en el master de Informática en la Universidad de La Coruña (Taboada, Touriño y Doallo, 2010), en Ingeniería de las telecomunicaciones de la Universidad Politécnica de Cataluña (Alcober, Ruiz y Valero, 2003), en el Grado de Ingeniería agroalimentaria y del medio rural de la Universidad Jaime I de Castellón (Pina y cols., 2015), en titulaciones como Pedagogía y Educación Social en la Universidad del País Vasco (Arandía y Fernández, 2012), o en la Universidad Católica de Valencia también en el grado de Educación Social (Guerrero y Calero, 2013), 


\section{APRENDIZAJE BASADO EN PROYECTOS EN EL ÁMBITO UNIVERSITARIO: UNA EXPERIENCIA DE INNOVACIÓN METODOLÓGICA EN EDUCACIÓN}

relacionando en los proyectos elaborados por los alumnos, las competencias académicas, la realidad social y las competencias laborales que necesitarán para desenvolverse exitosamente en su futuro profesional.

\section{MÉTODO}

\section{Participantes}

En el presente estudio participaron 228 estudiantes de la Facultad de Ciencias de la Educación de la Universidad de Granada, de los cuales 56 pertenecen al Grado de Pedagogía (alumnos de la asignatura Procesos y técnicas de intervención psicoeducativa), 119 al Grado de Educación Social (alumnos de la asignatura Psicología de la educación) y 53 al grado de Educación Infantil (alumnos de la asignatura Trastornos del desarrollo). De toda la muestra un $10.6 \%$ son hombres mientras que un $56.6 \%$ son mujeres. La edad media de la población es de 21.80 años.

\section{Instrumentos}

Se han utilizado para obtener los datos que se analizarán en la investigación dos cuestionarios. El primero de ellos se realiza durante las sesiones de tutoría, con el objetivo de conocer la evolución del grupo y de su trayectoria de trabajo.

* Cuestionario de Voluntad de Trabajo (Andrés et al., 2013): consta de 16 ítems con siete opciones de respuesta tipo Likert (1: nunca, 2: casi nunca, 3: rara vez, 4: pocas veces, 5: a veces, 6: bastante y 7: siempre). Presenta un alpha de Cronbach de .86. Los ítems de la escala se agrupan bajo una única dimensión, que mide aspectos motivacionales relacionados con la perseverancia y la responsabilidad. Los aspectos con los que se relacionan los ítems son la disposición al esfuerzo, la capacidad de automotivación frente a las obligaciones y los propios proyectos, el empeño en cumplir con las obligaciones aun contrariando el propio gusto, la inclinación a evadirse, el afrontar pronto el trabajo y el desarrollarlo con perseverancia.

* Cuestionario de Voluntad de Trabajo por Pares (Andrés et al., 2013): Presenta el mismo formato que el cuestionario anterior, la diferencia recae en que en vez de evaluarse la persona a sí misma, evalúa a terceros, a sus compañeros de grupo. El alpha de Cronbach es de .89 .

* Encuesta de evaluación de la satisfacción con la experiencia en la metodología de Aprendizaje basado en Proyectos (Rodríguez-Sandoval y Cortés-Rodríguez, 2010): Consta de 9 ítems, cuya respuesta es Sí o No.

\section{Procedimiento}

En el mes de octubre del curso académico 2015-2016, los estudiantes empezaron a trabajar en la experiencia del Aprendizaje Basado en Proyectos, eligiendo cada grupo un tema de los que se trabajarían en clase y realizando un proyecto práctico relacionado con el mismo. Posteriormente cumplimentaron los cuestionarios de Voluntad de Trabajo (individual y por pares), tras la primera tutoría grupal. La elaboración del proyecto se divide en varias fases:

Fase 1: Elección: El alumno elige el contenido de su proyecto de entre los diferentes temas que se trabajarán en las asignaturas, creando a partir del tema teórico un proyecto real. Reflexionarán sobre las necesidades e intereses de aprendizaje, lo cual hará que aumente su motivación intrínseca, sintiéndose protagonistas de la toma de decisiones. Se llevará a cabo un análisis de los conocimientos previos sobre cada tema, vinculándolos con las diferentes asignaturas.

Fase 2: Planificación: Es la fase de organización. Cada grupo se organizará las tareas en función de sus componentes y objetivos planteados. Introducirán en su planificación las tutorías grupales obligatorias, así como la fase de evaluación del proyecto. 
Fase 3: Desarrollo: es la fase de elaboración del proyecto propiamente dicha. En primer lugar harán una búsqueda de información, realizando un taller con Biblioteca para profundizar el aprendizaje sobre cómo llevar a cabo las revisiones bibliográficas. Posteriormente diseñarán el proyecto, siguiendo el guión previamente explicado. Partirán de una justificación del tema elegido, y de un análisis de necesidades, para centrarse en la descripción de la población a la que va dirigido, el objetivo de la intervención, los contenidos que se trabajarán en las diferentes actividades, la metodología de trabajo, temporalización y por último evaluación. Es importante que señalen qué competencias de las que deben adquirir en su formación universitaria, conseguirán al llevar a cabo el proyecto, así como qué conocimientos de otras asignaturas les han servido a la hora de realizarlo. El Ilevarlo a cabo o no en la práctica es voluntario, sí siendo obligatoria la exposición ante los compañeros.

Fase 4. Evaluación: La evaluación del proyecto se realizará de dos formas, la primera de manera individual, en la que cada alumno se autoevaluará en relación a las diferentes competencias trabajadas (mediante un cuestionario) y la segunda será una evaluación por pares. Cada alumno evaluará a los miembros de su grupo. Habrá una evaluación de desempeño, continua, en la que el profesor constatará los avances de los alumnos en las diferentes tutorías, y otra final, puntuando la memoria de proyecto presentada y la exposición pública al grupo clase.

Fase 5. Difusión: Se creará una página web en la que el alumnado puede subir y compartir los proyectos que ha llevado a cabo, a la vez que le dará acceso a toda la información y documentos que se vayan generando a lo largo del curso.

En la última tutoría grupal volverán a cumplimentar el Cuestionario de voluntad de Trabajo (Individual y por pares), en el mes de enero. Al finalizar las exposiciones de los trabajos y una vez que todo el procedimiento se ha llevado a cabo cumplimentarán el cuestionario de satisfacción con la experiencia de innovación.

\section{Análisis de datos}

Los datos fueron analizados con el paquete estadístico SPSS en su versión 20.0. Se realizaron comparaciones de media para muestras relacionadas ( $T$ de Student) en los cuestionarios de Voluntad de trabajo, con el objetivo de comparar los datos pre post, y se llevó a cabo un análisis de frecuencias para obtener los resultados que aportaban los datos del Cuestionario de Satisfacción.

\section{RESULTADOS}

A continuación, se exponen los datos obtenidos en el presente estudio. En una primera parte se recogen los datos del Cuestionario de Voluntad de Trabajo, tanto individual como de pares, y posteriormente se muestran los resultados obtenidos por el Cuestionario de Satisfacción.

\section{Resultados obtenidos tras el análisis del Cuestionario de Voluntad de Trabajo:}

En la Tabla 1 solamente se presentan los datos de los ítems cuyos resultados son significativos. Primero se estudiarán los datos del Cuestionario de Voluntad de Trabajo Individual, siendo significativos el ítem 2 Necesito que alguien me exija para cumplir bien con mis deberes $(\mathrm{t}(106)=.52$; $\mathrm{p}=.000)$, el 9 Dejo las cosas a medias $(\mathrm{t}(105)=-1.51 ; \mathrm{p}=.044)$, el 12 Suelo dejar para más tarde el cumplimiento de mis obligaciones ( $\mathrm{t}(107)=-.11 ; \mathrm{p}=.001)$, el 13 Prefiero saber un poco de distintos temas que mucho de algunos pocos $(\mathrm{t}(107)=1.28 ; \mathrm{p}=.020)$ y el 16 Cuando iba a la escuela, estudiaba como máximo lo imprescindible para aprobar $(\mathrm{t}(107)=.147 ; \mathrm{p}=.001)$. 
Tabla 1. Comparación de medias de los ítems del Cuestionario de Voluntad de Trabajo individual y por pares

\begin{tabular}{|c|c|c|c|c|c|c|}
\hline & & Media & $\mathbf{N}$ & Desv. & $\mathrm{T}$ & Sig. \\
\hline & & & & Típica & & \\
\hline \multirow[t]{3}{*}{ Item 2} & Pre & 2.45 & 107 & 1.494 & \multirow{3}{*}{.52} & \multirow{3}{*}{.000} \\
\hline & & & & & & \\
\hline & Post & 2.36 & 107 & 1.513 & & \\
\hline \multirow[t]{3}{*}{ Item 9} & Pre & 2.12 & 106 & 1.314 & \multirow{3}{*}{-1.51} & \multirow{3}{*}{.044} \\
\hline & & & & & & \\
\hline & Post & 2.38 & 106 & 1.411 & & \\
\hline \multirow[t]{3}{*}{ Item 12} & Pre & 2.72 & 108 & 1.497 & \multirow{3}{*}{-.11} & \multirow{3}{*}{.001} \\
\hline & & & & & & \\
\hline & Post & 2.74 & 108 & 1.494 & & \\
\hline \multirow[t]{3}{*}{ Item 13} & Pre & 4.27 & 108 & 1.378 & \multirow{3}{*}{1.28} & \multirow{3}{*}{.020} \\
\hline & & & & & & \\
\hline & Post & 4.06 & 108 & 1.277 & & \\
\hline \multirow[t]{3}{*}{ Item 16} & Pre & 3.17 & 108 & 1.710 & \multirow{3}{*}{.147} & \multirow{3}{*}{.001} \\
\hline & & & & & & \\
\hline & Post & 3.14 & 108 & 1.649 & & \\
\hline Item 2 & Pre & 2.49 & 108 & 1,476 & \multirow{3}{*}{-2.47} & \multirow{3}{*}{.001} \\
\hline \multirow[t]{2}{*}{ Pares } & & & & & & \\
\hline & Post & 2.94 & 108 & 1,745 & & \\
\hline Item 5 & Pre & 2.32 & 105 & 1,341 & \multirow{3}{*}{-2.79} & \multirow{3}{*}{.033} \\
\hline \multirow[t]{2}{*}{ Pares } & & & & & & \\
\hline & Post & 2.80 & 105 & 1,437 & & \\
\hline Item 6 & Pre & 5.55 & 108 & 1,500 & \multirow{3}{*}{.79} & \multirow{3}{*}{.029} \\
\hline \multirow{2}{*}{ Pares } & & & & & & \\
\hline & Post & 5.41 & 108 & 1,394 & & \\
\hline Item 7 & Pre & 3.31 & 107 & 1,514 & & \\
\hline Pares & & & & & -1.33 & .009 \\
\hline Pares & Post & 3.56 & 107 & 1,689 & & \\
\hline Item 9 & Pre & 2.04 & 101 & 1,232 & & \\
\hline Pares & & & & & -2.16 & .030 \\
\hline Fales & Post & 2.40 & 101 & 1,408 & & \\
\hline Item 11 & Pre & 2.44 & 106 & 1,455 & & \\
\hline Pares & & & & & -.59 & .008 \\
\hline Pares & Post & 2.55 & 106 & 1,519 & & \\
\hline Item 13 & Pre & 4.19 & 106 & 1,367 & & \\
\hline Pares & & & & & -.12 & 0.025 \\
\hline Pares & Post & 4.21 & 106 & 1,240 & & \\
\hline Item 16 & Pre & 3.03 & 104 & 1,657 & & \\
\hline Pares & & & & & .21 & .010 \\
\hline & Post & 2.99 & 104 & 1,376 & & \\
\hline
\end{tabular}


En cuanto a la Voluntad de Trabajo por Pares, el ítem 2 de nuevo es significativo Necesito que alguien me exija para cumplir bien con mis deberes $(\mathrm{t}(107)=.-2.47 ; \mathrm{p}=.001)$, el 5 Se ocupan más de terminar el trabajo cuanto antes que de hacerlo bien ( $\mathrm{t}(104)=-2.79 ; \mathrm{p}=.033)$, el 6 Aunque se sientan cansados terminan con la tarea que se impusieron $(\mathrm{t}(107)=.79 ; \mathrm{p}=.029)$, el 7 Dan muchas vueltas antes de ponerse a trabajar $(\mathrm{t}(106)=-1.33 ; \mathrm{p}=.009)$, el 9 Dejo las cosas a medias $(\mathrm{t}(100)=-2.16$; $\mathrm{p}=.030)$, el 11 Cualquier excusa les viene bien para interrumpir el trabajo $(\mathrm{t}(105)=-.59 ; \mathrm{p}=.008)$, el 13 Prefiero saber un poco de distintos temas que mucho de algunos pocos $(\mathrm{t}(105)=-.12 ; \mathrm{p}=.025)$ y el 16 Cuando iba a la escuela, estudiaba como máximo lo imprescindible para aprobar $(\mathrm{t}(103)=.21$; $\mathrm{p}=.010)$.

Resultados obtenidos tras el análisis del Cuestionario de Satisfacción:

A continuación se presentan los datos obtenidos del Cuestionario de Satisfacción, siendo "Si" un 1 y "no" un 2. En el ítem 1 ¿Cree usted que el proyecto de aula es importante para su formación profesional? el 100\% de la muestra señala que sí es importante. En el ítem 2 ¿Fue difícil encontrar una idea de proyecto apropiado para desarrollar en el curso? Un 81.6 de los estudiantes señalan que no $(\mathrm{M}=1.82 ; \mathrm{DT}=.389)$. El ítem 3 ¿Se aplicaron los conceptos vistos en clase para la solución de los problemas del proyecto de aula? es puntuado por $92.8 \%$ de los alumnos de forma positiva $(\mathrm{M}=1.07$; $\mathrm{DT}=.260$ ). En el ítem 4 ¿Cree usted que la forma de realizar el proyecto durante el semestre fue la adecuada? Un $87 \%$ piensa que sí ( $\mathrm{M}=1.13$; $\mathrm{DT}=.338$ ). Un $94.9 \%$ piensan que están conformes con la forma de realizar los avances (tutorías de seguimiento) del proyecto $(\mathrm{M}=1.05 ; \mathrm{DT}=.220)$. Un $71 \%$ cree que el tiempo estipulado para el desarrollo del proyecto es suficiente $(\mathrm{M}=1.29$; $\mathrm{DT}=.455)$.

Figura 1. Porcentaje de respuesta en el Cuestionario de Satisfacción

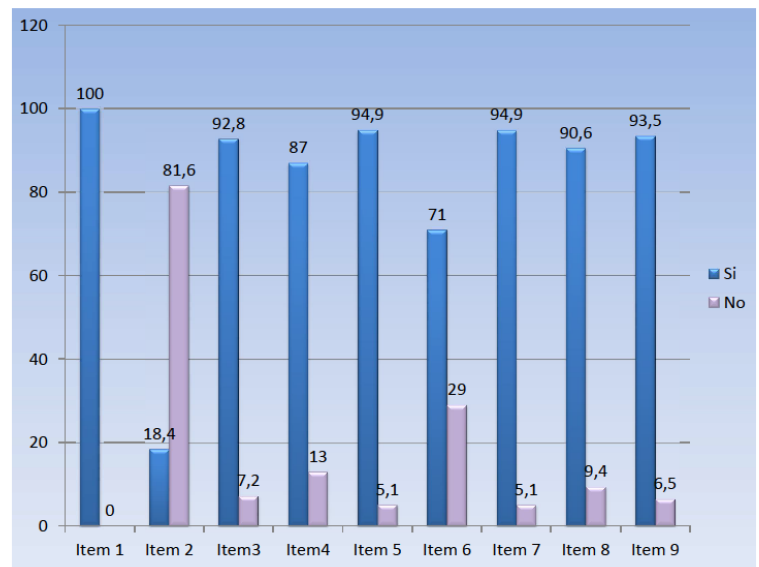

El ítem 7 ¿Este tipo de metodologías para el aprendizaje satisfacen sus expectativas como estudiante comparándola con otros métodos tradicionales? lo puntúa un $94.9 \%$ de alumnos de forma positiva ( $\mathrm{M}=1.05$; $\mathrm{DT}=.220)$. En el ítem 8¿Considera que los materiales y demás recursos disponibles, son adecuados para el desarrollo del proyecto? un $90.6 \%$ de los estudiantes creen que son adecuados ( $\mathrm{M}=1.09$; $\mathrm{DT}=.293)$ y por último, un $93.5 \%$ del alumnado Considera que la orientación para el desarrollo del trabajo por parte del docente cumplió con sus expectativas $(\mathrm{M}=1.07$; $\mathrm{DT}=.248)$. 


\section{APRENDIZAJE BASADO EN PROYECTOS EN EL ÁMBITO UNIVERSITARIO: UNA EXPERIENCIA DE INNOVACIÓN METODOLÓGICA EN EDUCACIÓN}

\section{DISCUSIÓN Y CONCLUSIONES}

En relación a la voluntad de trabajo, trabajos previos señalan que los alumnos se perciben a sí mismos como responsables y perseverantes, con una actitud positiva frente al trabajo (Galibert, Abal, Lozzia, Aguerri y Attoresi, 2006). Presentan una cierta sobreestimación de su desempeño, lo cual quizás puede deberse a la deseabilidad social, 0 a la estimación subjetiva que realizan de su propio esfuerzo.

Comparando los resultados del cuestionario individual y del de pares, se observa que los estudiantes son más críticos con la voluntad de trabajo de sus compañeros que con la de ellos mismos, por ejemplo, se ven más responsables que el resto. Esta situación se constata en el presente estudio, ya que opinan que conforme pasa el cuatrimestre necesitan en menor medida que alguien les exija para cumplir con su trabajo, lo contrario que opinan de los compañeros de grupo, aumentando de forma significa las medias en la post evaluación. Piensan que sus compañeros a veces se preocupan más de terminar que de tener resultados positivos en la elaboración del proyecto, y aunque ellos creen que dejan las cosas a medias, y a veces para el final, lo cierto es que creen que sus iguales lo hacen en mayor medida. Por lo que existe una percepción más positiva de la voluntad de trabajo propia, destacando una mayor motivación y perseverancia, que de la de los demás.

En cuanto a la satisfacción del alumnado con la implementación de la metodología del ABPr, los resultados encontrados en la presente investigación son acordes con los que se obtienen en diferentes estudios. Reverte, Gallego, Molina y Satorre (2006), encuentran que el uso de esta metodología reporta al alumnado más satisfacción y aumenta su interés, no resultándoles difícil llevarla a cabo. Los alumnos valoraron positivamente el relacionar los contenidos teóricos con su futuro profesional, aplicándolos a la realidad. Describen como satisfactorio el trabajo en grupo (aspecto que también destacan Alcober, Ruiz y Valero en el 2003) y su labor de investigación, aunque la búsqueda de material no les resulta del todo fácil. Además, obtienen mejores calificaciones y recuerdan lo aprendido durante más tiempo que utilizando una metodología tradicional (Mioduser y Betzer, 2007). Activan conocimientos previos, mejorando la metacognición y las habilidades de resolución de problemas (Guerrero y Calero, 2013).

Comparando los resultados del presente estudio con los de Rodríguez y Cortés (2009), quienes utilizan el mismo cuestionario de satisfacción, se observa que en ambos, el 100\% del alumnado está satisfecho con la experiencia. El $57 \%$ de los alumnos de dicho estudio consideran que fue difícil encontrar un tema, sin embargo el $81.6 \%$ del nuestro creen que no, quizás sea porque las temáticas se ceñían a los temas de la asignatura. También es, en ambos trabajos, elevado el número de estudiantes que opinan que el procedimiento se llevó a cabo de forma apropiada aunque piensan, en un $71 \%$, que el tiempo no es adecuado, solicitando más tiempo para diseñar los proyectos. Piensan que son positivas las tutorías de seguimiento y la labor que hace el docente. En un $94 \%$ creen que es mejor este método que el llevar a cabo en las clases una metodología tradicional.

Pero aunque tanto profesores como alumnos valoran dicha experiencia, no está exenta de limitaciones, ya que requiere un mayor seguimiento personalizado y una mayor interrelación con el alumnado por parte del profesorado, que si bien es muy positivo incrementa el trabajo y el tiempo de dedicación (Estruch y Silva, 2006), debiendo estar muy estructurado todo el proceso. Los mismos autores señalan otro aspecto como limitación, siendo éste las dificultades que a veces tienen los estudiantes para trabajar en grupo.

Lo que sí queda confirmado es que el ABPr minimiza las limitaciones de la docencia tradicional, fomentando habilidades como son el trabajo en grupo, la planificación del tiempo, el trabajo autónomo, la capacidad de expresión oral y escrita, las habilidades intelectuales de alto nivel y la autoevaluación (Reverte, Gallego, Molina y Satorre, 2006), siendo factible en una gran variedad de asig- 
naturas y en diferentes áreas de conocimiento (García y Amante, 2006), aumentando el rendimiento del alumno.

\section{REFERENCIAS BIBLIOGRÁFICAS}

Alcober, J., Ruiz, S., Valero, M. (2003). Evaluación de la implantación del aprendizaje basado en proyectos en la EPSC (2001-2003). XI Congreso Universitario de Innovación Educativa en las Enseñanzas Técnicas, Vilanova i la Geltrú.

Andrés, A., Abal, F., Lozzia, G., Gómez-Benito, J., Aguerri, M., Galibert, M. y Attorresi, H. (2013). Adaptación del cuestionario de "Voluntad de Trabajo" a una muestra de universitarios españoles. Anales de Psicología, 29 (1), 171-177.

Arandia, M. y Fernández, I. (2012). ¿Es posible un curriculum más allá de las asignaturas? Diseño y práctica del grado de Educación Social en la Universidad del País Vasco. Revista de docencia universitaria, 10 (3), 99-123.

Carrasco, A., Donoso, J. A., Duarte-Atoche, T., Hernández, J. J. y López, R. (2015). Diseño y validación de un cuestionario que mide la percepción de efectividad del uso de metodologías de participación activa (CEMPA). El caso del aprendizaje basado en proyectos (ABPrj) en la docencia de la contabilidad. Innovar, 25 (58), 143-158.

Collazos, C. (2009). Enseñanza de la conservación del momento angular por medio de la construcción de prototipos y el aprendizaje basado en proyectos. Latin-American Journal of Physics Education, 3 (2), 427-432.

Estruch, V. y Silva, J. (2006). Aprendizaje basado en proyectos en la carrera de Ingeniería informática. JENUI, 339-346.

Galibert, M., Abal, F., Lozzia, G., Aguerri M. y Attoresi, H. (2006). Voluntad de trabajo en estudiantes de psicología. Anuario de investigaciones, 14.

García-Almiñana, D. y Amante, B. (2006). Algunas experiencias de aplicación del aprendizaje cooperativo y del aprendizaje basado en proyectos. I Jornadas de Innovación Educativa. Zamora: Escuela Politécnica Superior de Zamora.

Guerrero, E. y Calero, J. (2013). El aprendizaje basado en proyectos como base metodológica en el Grado de Educación Social. Educación Social. Revista de intervención socioeducativa, 53. 7391.

Mioduser, D. y Betzer, N. (2007). The contribution of Project-based learning to high achievers acquisition of technological knowledge. International Journal of technology and design education, 18, 59-77.

Muñoz, A. y Díaz, M. R. (2009). Metodología por proyectos en el área de conocimiento del medio. Revista docencia e investigación, 19, 101-126.

Navarro, I., Pertegal, M., Gil, D., González, C. y Jimeno, A. (2011). El aprendizaje basado en proyectos como estrategia didáctica y pedagógica para estimular el desarrollo de competencias profesionales. IX Jornadas de redes de investigación en docencia universitaria.

Nourdine, A. y Bemposta, S. (2008). Una experiencia de aprendizaje basado en proyectos en una asignatura de robótica. IEEE Revista Iberoamericana de Tecnologías del Aprendizaje, 3 (2), 7176.

Pina, T., Aguilar, E., Camañes, G., Marqués, A., Rodríguez, A., Fernández, E., Llorens, E., Scalschi, L., Máñez, M., Jacas, J., Vicedo, B. y Lapeña, L. (2015). Implementation of a project-based learning to the coordination of subjects in the Agrifood and rural engineering bachelor. Revista de docencia universitaria, 13 (3), 265-287.

Reverte, J. R., Gallego, A. J., Molina, R. y Satorre, R. (2006). El aprendizaje basado en proyectos 
como modelo docente: experiencia interdisciplinar y herramientas groupware. Proyecto de innovación tecnológico-educativo e innovación educativa de la Universidad de Alicante.

Rodríguez-Sandoval, E. y Cortés-Rodríguez, M. (2010). Evaluación de la estrategia pedagógica "Aprendizaje basado en proyectos". Percepción de los estudiantes. Avaliação (Campinas; Sorocaba), 15 (1), 143-158.

Sánchez, M. y Vidal, 0. (2013). Aprendizaje colaborativo basado en proyectos desarrollados en ingeniería. Revista iberoamericana para la investigación y el desarrollo educativo, 10, 1-15.

Taboada, G., Touriño, J. y Doallo, R. (2010). Innovación docente en el EEES de cara a la práctica profesional a través del aprendizaje basado en proyectos. XVI Jornadas de enseñanza universitaria de la informática. Santiago de Compostela. 